Noman 2019, 37(1), 35-42

Revista de Psicologia, Ciències de l'Eduació i de l'Esport

ISSN: $1138-3194$

CFacultat de Psicologia, Ciències de l'Educació i de l’Esport Blanquerna

Universitat Ramon Llull

\title{
Tinder y conductas sexuales de riesgo en jóvenes españoles
}

\author{
Oscar $\mathrm{Wu}$ \\ Universidad Autónoma de Barcelona
}

Received: 13 feb 2019

Accepted: 1 apr 2019

Tinder y conductas sexuales de riesgo en jóvenes españoles

Resumen. La popularidad de las apps móviles con geolocalización permite ampliar el círculo social de muchas personas, pero existen escasas investigaciones acerca del impacto que puedan tener sobre la salud de los usuarios. Tinder es la app con geolocalización más popular y resulta conveniente analizar cómo afecta a la sexualidad de los usuarios y si puede propiciar conductas de riesgo. El objetivo de la presente investigación es determinar los efectos del uso de Tinder: la búsqueda de sensaciones sexuales, compulsividad sexual y conductas sexuales de riesgo, como el sexo sin preservativo, sexo con múltiples parejas y sexo no consentido. Participaron 262 sujetos de entre 18 y 30 años, de los cuales 133 eran usuarios de Tinder y 129 no usuarios. Los usuarios de Tinder obtuvieron puntuaciones mayores en búsqueda de sensaciones sexuales y compulsividad sexual que los no usuarios. No hubo diferencias en las conductas sexuales de riesgo, salvo que los usuarios de Tinder hacen un mayor uso del preservativo. En conclusión, pese a puntuar más alto en compulsividad sexual y búsqueda de sensaciones sexuales, los usuarios de Tinder tienen sexo más seguro que los no usuarios porque presentan mayor uso del preservativo y no hay diferencias en el resto de conductas sexuales de riesgo.

Palabras clave: Tinder; búsqueda de sensaciones sexuales; compulsividad sexual; conductas sexuales de riesgo

\section{Tinder and risky sexual behaviour in young Spaniards}

Summary. Location-based mobile apps have had an impact on the lives of many of their users, allowing them to widen their social circles, but there have been few publications regarding the effect these apps may have on users' health. Tinder is the most well-known location-based mobile app, and as it continues to gain users, the question of how it affects its users' sexuality and of whether it might prompt them to engage in risky sexual behaviours are topics worthy of investigation. The aim of this research is to determine the effects of Tinder use: sexual sensation seeking, sexual compulsivity and risky sexual behaviours such as intercourse without condoms, intercourse with multiple partners and intercourse without consent. 262 subjects from Barcelona with ages ranging from 18-30 participated in the study. 133 of them were Tinder users and 129 were non-users. Tinder users recorded higher scores for sexual sensation seeking and sexual compulsivity than non-users. No differences were found regarding risky sexual behaviors, except that Tinder users use condoms more frequently than non-users. In conclusion, despite having higher scores for sexual compulsivity and sexual sensation seeking, Tinder users have safer sexual intercourse than non-users, due to their greater use of prophylaxis and the lack of significant differences in the other risky sexual behaviors.

Keywords: Tinder; sexual sensation seeking; sexual compulsivity; risky sexual behaviors 


\section{Introducción}

Más del 85\% de los jóvenes de entre 18 y 29 años tienen un smartphone con acceso a Internet, y el intercambio de mensajes mediante esta vía es casi universal entre los jóvenes (Smith, 2015). De este modo, el smartphone brinda a sus usuarios, entre sus múltiples funciones, una gran facilidad para comunicarse con otros y casi constante conectividad. Como consecuencia, los recursos digitales influencian también el modo en que la población, mayoritariamente la joven, se comunica, y por extensión busca pareja y relaciones íntimas.

El fenómeno de usar las TIC para conocer nuevas posibles parejas sentimentales se denomina citas online. En la primera década del siglo xx hubo un auge de las webs de citas "basadas en la ciencia", en las que un algoritmo presentaba múltiples perfiles de personas compatibles con el usuario basándose en una exhaustiva recogida de información para encontrar parejas románticas. La creciente popularidad de las citas online conllevó que en 2009 en Estados Unidos se iniciaran más relaciones románticas mediante los recursos digitales que de ningún otro modo, salvo las parejas que se conocieron siendo presentadas por amistades en común (Finkel, Eastwick, Karney, \& Reis, 2012). Una ventaja de las citas online es que propician oportunidades de acceder a nuevas relaciones. Además, investigaciones recientes apuntan a que el hecho de poder emprender una breve interacción inicial mediada por las TIC con una posible pareja tiene el potencial de suscitar mayor atracción antes del encuentro en persona (Finkel et al., 2012).

Las numerosas webs de citas "basadas en la ciencia" han sido desbancadas entre los jóvenes por apps móviles, frecuentemente descritas como más rápidas y requeridoras de poco esfuerzo (Timmermans \& De Caluwé, 2017a). En contraste con sus predecesoras, dichas apps no recopilan un volumen tan grande de información, pero cuentan con el atractivo añadido de la geolocalización. De este modo, a los usuarios se les presentan una serie de perfiles cercanos a ellos y se crea una interacción que va más allá de lo online, e incentiva a los usuarios a verse en la "vida real" (Cohen, 2015; Gibbs, Ellison, \& Lai, 2011). Con la geolocalización ya no se trata de una realidad virtual y una física independientes entre sí, sino que el uso de las apps se entendería como un modo de establecer conexiones en tiempo real con personas cercanas, de modo similar al funcionamiento de la realidad aumentada, teniendo disponibles para interactuar a personas que no se encuentran físicamente delante del usuario, pero pudiendo establecer una relación con ellas (Jurgenson, 2012).

Así como los adultos de edad media tienden más a buscar relaciones serias y a largo plazo, entre los jóvenes adultos es más probable que vayan entrando y saliendo de relaciones, viéndose a menudo inmersos en relaciones casuales (Cohen, Kasen, Chen, Hartmark, \& Gordon, 2003). Por un tema de comodidad, estas relaciones casuales son más fáciles de encontrar en apps con geolocalización, ya que se busca principalmente proximidad y atracción física. Adicionalmente, Sprecher y Sprecher (2009) hallaron que en entornos digitales las personas están más abiertas a seleccionar o encontrar pareja. No obstante, en estudios llevados a cabo con jóvenes de entre 18 y 30 años, un tercio de ellos dejaron de usar estas apps porque habían iniciado una relación con alguien conocido mediante ellas (Newett, Churchill, \& Robards, 2017), de modo que un porcentaje relativamente amplio de los usuarios usan las apps para establecer relaciones más allá del contacto físico casual. Pese al aumento en el número de usuarios, existen publicaciones como la de Smith y Anderson (2016) en las que nos informan que hasta un tercio de los americanos usuarios de estas tecnologías no habían tenido ninguna cita en persona con alguien conocido en una página web de citas o en una $a p p$ móvil.

Entre estas apps con geolocalización destaca Tinder, con más de 50 millones de usuarios, la mayoría de los cuales son jóvenes adultos de edades comprendidas entre los 18 y los 24 años. Tinder, siendo de base gratuita, tiene opciones prémium y es la app que más beneficios económicos reporta (Sales, 2015). Según Newett et al. (2017), Tinder era una alternativa accesible para conectar con otras personas, y sirve para combatir las dificultades que los jóvenes tienen para establecer relaciones de pareja, como la necesidad de encontrarla gracias a un grupo de amigos o en el trabajo, pudiéndolo hacer desde la comodidad de su casa. Para crear un perfil en Tinder, el usuario debe mostrar una selección de entre 1 y 6 fotografías, y especificar su edad y género. Adicionalmente, se puede vincular la cuenta con Facebook, Instagram y Spotify para verificar la edad y disponer más fácilmente de algunas fotografías e información adicional como gustos musicales o amistades en común que se puedan tener con otros usuarios. A continuación, se puede publicar una breve descripción del usuario, con un máximo de 500 caracteres. Una vez el perfil está creado, se seleccionan las preferencias de búsqueda: hombres, mujeres o ambos, el rango de edad que se prefiera y la distancia máxima en kilómetros. Cuando esta serie de ajustes están configurados, al usuario se le van presentando una serie de perfiles de personas que cumplen con sus criterios de búsqueda, y este deberá ir aceptándolos o descartándolos de forma anónima. La finalidad de Tinder es que cuando se acepta un perfil y la otra persona hace lo mismo, se abre un chat privado para iniciar la interacción. Se denomina match cuando dos usuarios manifiestan interés por el perfil del otro, y cada match precede a la apertura del chat entre ellos. Por lo tanto, Tinder actúa como un "intermediario" en la vida íntima de los usuarios proporcionando la posibilidad de ampliar horizontes relacionales (Newett et al., 2017).

La diferencia más relevante de Tinder respecto a las webs de citas "basadas en la ciencia" es que no consta de ninguna descripción detallada de la persona más allá de los 500 caracteres, ni de ningún algoritmo de 
compatibilidad. Esto hace que las elecciones de potenciales parejas se vean influenciadas principalmente por la apariencia física, cosa que puede hasta llevar a que los usuarios cosifiquen a las personas que se les presentan (Finkel et al., 2012; Timmermans, \& De Caluwé, 2017b). Con la relativa escasez de información proporcionada en los perfiles de los usuarios, se enfatiza la importancia de las claves visuales en las fotografías, que influencia la toma de decisiones (Ranzini \& Lutz, 2017). La priorización del aspecto físico, sin embargo, no implica que se reste importancia a la autopresentación escrita, pues diversas investigaciones han concluido que tanto hombres como mujeres tienden a preferir los perfiles en los que hay una descripción personal (Ranzini \& Lutz, 2017). Heino, Ellison y Gibbs (2010) indicaron que la naturaleza y la estructura comercial de las apps como Tinder llevaban a los usuarios a racionalizar, priorizando variables como la proximidad o los aspectos físicos por encima de los sentimientos al elegir pareja, resultando en elecciones que de otro modo serían poco probables. Al no conocer a la mayoría de los otros usuarios de Tinder, la persona tiene el poder de maquillar su perfil con fotos expresamente seleccionadas o hasta mintiendo en la descripción, restándole realismo. Ranzini y Lutz (2017) hallaron que las personas con mayor autoestima y que buscaban establecer una relación sentimental se mostraban de un modo más realista en su perfil de Tinder, y que una autopresentación artificial o engañosa estaba significativamente influenciada por la autoestima, nivel educativo y orientación sexual. De este modo, los usuarios con mayor nivel educativo, homosexuales y bisexuales tendían a ser menos realistas al presentarse, y las principales razones por las que usaban Tinder eran conseguir relaciones sexuales y autovalidación.

Dada la popularidad de Tinder, desde una perspectiva psicológica, la investigación ha explorado los motivos y las preocupaciones derivados de su uso. Estudios recientes en muestras americanas (Griffin, Canevello, \& Mcanulty, 2018) exponen que las principales razones para usarlo son "por diversión" y para "conocer gente", y aunque es menos probable que los usuarios inicien una relación sentimental, contrasta con el estigma generado por los medios, que tilda la app como la app del sexo casual. Aunque las personas conocidas mediante Tinder puedan resultar en una gran variedad de tipos de relación, incluyendo platónicas y románticas, diversos medios de comunicación describen la app como de naturaleza predominantemente sexual, usada por jóvenes para "tener sexo sin compromiso" (Sales, 2015). El uso "por diversión" se relaciona directamente con buscar estimulación y combatir el aburrimiento. Tinder se puede utilizar cómodamente en el transporte público, de camino a casa, escuchando música o incluso en presencia de amistades con quienes ir comentado los distintos perfiles que van apareciendo.

Green, Turner, y Logan (2018) exploraron el efecto que las amistades en común de Facebook tenían sobre las conductas sexuales de riesgo en los usuarios de Tinder, y encontraron que las amistades en común precipitaban conductas sexuales de riesgo, tales como sexo sin profilaxis y no hablar de enfermedades de transmisión sexual. La tendencia a asumir estos riesgos se explicaba por el temor a que la información que se compartiera pudiera llegar a oídos de las amistades en común y se percibía a la otra persona como "familiar", dando una falsa imagen de seguridad acerca de posibles riesgos para la salud. Por lo tanto, esta falsa sensación de seguridad, unida a otros factores de riesgo como el consumo de alcohol y una alta búsqueda de sensaciones sexuales (Gullette \& Lyons, 2006), podría llevar al desarrollo de conductas sexuales de riesgo. Sin embargo, en un estudio con estudiantes universitarios, Ingram, Macauda, Lauckner, y Robillard (2018) hallaron que los jóvenes solteros tendían a usar preservativos con mayor frecuencia que los que estaban en una relación de pareja, y una amplia mayoría de los usuarios de Tinder lo usan sin estar en una relación sentimental.

Entre las conductas sexuales de riesgo más relevantes se encuentra la de tener relaciones con múltiples parejas. Si bien el uso de Tinder no implica ausencia de protección a la hora de tener relaciones sexuales, sí que posibilita un modo adicional de encontrar personas con quien tener relaciones. Estas mayores opciones de pareja también ofrecen más posibilidades para la "traición" o infidelidad (Murray \& Campbell, 2015) y la capacidad de mantener múltiples relaciones simultáneamente. Las otras dos grandes conductas sexuales de riesgo investigadas en relación con Tinder han sido: no usar preservativos y tener relaciones sexuales no consentidas, y se encontró que el uso de Tinder se correlaciona significativamente con la segunda (Shapiro et al., 2017). Para el presente estudio, se ha considerado conveniente añadir una conducta sexual de riesgo adicional más allá de las mencionadas anteriormente: tener relaciones sexuales sin estar seguro de querer tenerlas. Fox y Moreland (2015) sugirieron que los participantes que tenían un uso más intensivo de Facebook podían sentirse presionados a mantener relaciones que no querían.

El presente estudio se propone investigar la influencia que pueda ejercer Tinder a la hora de adoptar conductas sexuales de riesgo entre sus usuarios. Las hipótesis son:

1. Los usuarios de Tinder tienen más compulsividad sexual y búsqueda de sensaciones sexuales que los no usuarios (Gil-Llario, Morell-Mengual, BallesterArnal, Giménez-García, \& Castro-Calvo, 2015).

2. Los usuarios de Tinder mostrarán más conductas sexuales de riesgo (sexo sin preservativo, sin consentimiento y con múltiples parejas) que los no usuarios (Shapiro et al., 2017).

3. Los usuarios problemáticos de Tinder puntuarán más alto en búsqueda de sensaciones sexuales, compulsividad sexual y conductas sexuales de riesgo que los usuarios no problemáticos (BallesterArnal et al., 2015; Gil-Llario et al., 2015; Shapiro et al., 2017). 


\section{Método}

\section{Participantes}

Participaron 273 sujetos, pero se desestimaron 11 sujetos por no haber completado correctamente los cuestionarios o no cumplir con los parámetros de edad requeridos (rango de edad entre 18 y 30 años). Los 262 restantes presentaban una media de edad de 23,14 años (DT $=2.11$ ). El 43.1\% eran hombres y el 56.9\% mujeres. Un total de 133 sujetos fueron usuarios de Tinder (50.8\%) y 129 no usuarios (49.2\%).

\section{Instrumentos}

Búsqueda de sensaciones sexuales. Para evaluar la búsqueda de sensaciones sexuales se ha usado la Sexual Sensation Seeking Scale (Kalichman, Johnson, Adair, Rompa, \& Kelly, 1994) adaptada al castellano (GilLlario et al., 2015). El instrumento mide la tendencia a buscar nuevas experiencias y prácticas sexuales con ítems como "Me gustan las sensaciones y experiencias sexuales nuevas y excitantes". Se trata de una versión de 10 ítems de respuesta tipo Likert, que van desde 1 (Nada característico de mí) hasta 4 (Muy característico de mí). En el presente estudio, el Alfa de Cronbach obtenido ha sido de .72 .

Compulsividad sexual. Se ha usado la Sexual Compulsivity Scale (Kalichman, Johnson, Adair, Rompa, \& Kelly, 2010) en su versión española (Ballester-Arnal, Gómez-Martínez, Gil-Llario, \& Salmerón-Sánchez, 2013). Se trata de un cuestionario Likert de 10 ítems que van desde 1 (Nada característico de mí) hasta 4 (Muy característico de mí). Mide preocupaciones obsesivas acerca de actos y encuentros sexuales, como por ejemplo: "Mis pensamientos y comportamientos sexuales me están causando problemas en la vida". El Alfa de Cronbach obtenido ha sido de .85.

Uso problemático de Tinder. Se ha medido usando la versión de 6 ítems de la Problematic Tinder Use Scale (Orosz, Tóth-Király, Bőthe, \& Melher, 2016), traducida ad hoc para la presente investigación. Consta de 6 ítems en escala Likert que oscilan entre 1 (Nada característico de mí) hasta 5 (Muy característico de mí), y se basa en el modelo de los seis componentes de adicción de Griffiths: relevancia, tolerancia, modificación de humor, recaída, abstinencia y conflicto. Se ha obtenido un Alfa de Cronbach de .78. Son ejemplos de ítems: "Durante el último año, ¿con qué frecuencia ha usado Tinder para reducir sentimientos de culpa, ansiedad, indefensión y depresión?" y "Durante el último año, ¿con qué frecuencia ha intentado reducir su uso de Tinder sin éxito?".

Uso de Tinder. Se presentaron ítems relativos a la frecuencia y duración de las conexiones, como: "Aproximadamente, ¿cuántas veces al día entra a Tinder?", "¿Aproximadamente, cuánto duran sus conexiones a Tinder?”, y búsqueda de pareja: “¿Con qué porcentaje de matches de Tinder ha quedado en persona?" y "Entre los matches de Tinder con los que llegó a verse en persona, ¿con cuántos mantuvo relaciones sexuales?".

Conductas sexuales de riesgo. Para evaluar si se dan conductas sexuales de riesgo en los sujetos, se han incluido las siguientes preguntas contabilizadas por separado: "Normalmente, ¿usa preservativo al tener relaciones sexuales?" (Likert 4: "siempre, casi siempre, casi nunca, nunca"), "En el último mes, ¿cuántas parejas sexuales ha tenido?" (Likert 5: "ninguna, 1, 2, 3 o 4, más de 4") y “¿Ha tenido alguna vez relaciones sexuales no consentidas por su parte?" (de respuesta dicotómica). A dichas preguntas se ha añadido adicionalmente "¿Ha tenido alguna vez relaciones sexuales sin estar seguro de querer tenerlas?" (de respuesta dicotómica). Estos ítems han sido acompañados de las preguntas: "Normalmente, ¿con qué frecuencia mantiene relaciones sexuales?" (Likert 4: "0 veces a la semana, 1-2, 3-4 y más de 4"), “Usó preservativo la última vez que mantuvo relaciones sexuales?" (de respuesta dicotómica) y "Normalmente, ¿usa algún anticonceptivo que no sea el preservativo cuando mantiene relaciones sexuales?" (de respuesta dicotómica).

\section{Procedimiento}

Se creó un formulario de Google que incluía los $3 \mathrm{cu}$ estionarios y una serie de ítems para recopilar datos demográficos, explorar conductas sexuales de riesgo y uso de Tinder. Se accedió a la muestra mediante difusión por WhatsApp y Facebook, y siguiendo un muestreo de bola de nieve. Al inicio del formulario, se incluyó un consentimiento informado, de modo que todos los sujetos dieron su consentimiento para usar los datos personales recopilados de manera anónima. Para el análisis de datos, se utilizó la versión 24 del programa IBM SPSS Statistics.

\section{Análisis de datos}

Para explorar los efectos del uso de Tinder y el sexo de los sujetos, se efectuaron ANOVAS 2x2 (sexo x uso de Tinder) para las variables: uso de preservativo, sexo con dudas, sexo no consentido, búsqueda de sensaciones sexuales y compulsividad sexual. Las variables "sexo no consentido" y "sexo con dudas", al ser dicotómicas, han sido analizadas mediante la prueba chi-cuadrado. Se calcularon correlaciones de Pearson para analizar la relación del uso problemático de Tinder, matches conocidos en persona, matches con los que se ha tenido relaciones sexuales, minutos por conexión a Tinder, número de conexiones diarias a Tinder, compulsividad sexual y búsqueda de sensaciones sexuales.

\section{Resultados}

La puntuación media de búsqueda de sensaciones sexuales fue de 26,16, con una desviación típica de 5,03. En compulsividad sexual, la media fue de 14,24 y la desviación típica 4,64. Los estadísticos descriptivos de uso del preservativo, parejas sexuales al mes, sexo con 
Tabla 1. Medias y desviaciones típicas (entre paréntesis) de las variables estudiadas

\begin{tabular}{|c|c|c|c|c|}
\hline \multirow[b]{2}{*}{ Variable } & \multicolumn{2}{|c|}{ Uso de Tinder } & \multicolumn{2}{|c|}{ No uso de Tinder } \\
\hline & Hombres & Mujeres & Hombres & Mujeres \\
\hline Uso del preservativo & $2.81(.87)$ & $2.72(.94)$ & $2.27(1.23)$ & $2.12(1.18)$ \\
\hline Parejas sexuales al mes & $2.04(.95)$ & $2.12(.92)$ & $2.22(.704)$ & $1.89(.44)$ \\
\hline Sexo con dudas & $1.50(.50)$ & $1.54(.50)$ & $1.33(.477)$ & $1.40(.49)$ \\
\hline Sexo no consentido & $1.06(.24)$ & $1.11(.31)$ & $1.07(.252)$ & $1.10(.29)$ \\
\hline Búsqueda de sensaciones sexuales & $28.59(3.84)$ & $25.83(5.60)$ & $26.96(4.76)$ & $24.04(4.64)$ \\
\hline Compulsividad sexual & $16.03(4.71)$ & $14.26(5.10)$ & $14.49(4.79)$ & $12.65(3.53)$ \\
\hline
\end{tabular}

dudas, sexo no consentido, búsqueda de sensaciones sexuales y compulsividad se muestran en la Tabla 1, separados por sexo y uso de Tinder.

En la primera de las conductas sexuales de riesgo, 22 sujetos $(8.4 \%)$ declararon haber tenido relaciones sexuales sin haber dado su consentimiento. En la segunda conducta sexual de riesgo, 69 sujetos (26.3\%) no usaban nunca el preservativo, 53 (20.2\%) no lo usaban "casi nunca", 87 (33.2\%) lo usaban "casi siempre" y 53 (20.2\%) aseguraban usar siempre el preservativo. En lo relativo a la última conducta sexual de riesgo, mantener relaciones sexuales con múltiples parejas, 51 sujetos (19.5\%) no habían tenido ninguna pareja sexual en el último mes, 165 (63\%) habían tenido una pareja sexual, 34 (13\%) habían tenido dos, 7 $(2.7 \%)$ habían tenido entre tres y cuatro, y 5 sujetos (1.9\%) habían tenido relaciones con más de cuatro parejas distintas.

118 sujetos (45\%) habían tenido relaciones sexuales con dudas acerca de si querer tenerlas, mientras que 144 (55\%) no las habían tenido.

No hay diferencias significativas entre usuarios y no usuarios a la hora de tener relaciones sexuales no consentidas. Además, de los 22 sujetos que las habían tenido, 11 eran usuarios de Tinder y 11 no lo eran. No obstante, con el valor se demuestra que sí hay diferencias significativas en lo referente a tener relaciones sexuales sin estar seguro de querer tenerlas $\left(\chi^{2}=5.19\right.$; $p=.002$ ). Un $45.04 \%$ de los sujetos de estudio han mantenido relaciones sexuales teniendo dudas al respecto; 69 son usuarios de Tinder y 49 no usuarios.

El análisis multivariado de la varianza para los efectos del uso de Tinder y sexo en el uso del preservativo (ver Tabla 2) indica que no hay diferencias entre sexos, pero sí según el uso de Tinder, de tal forma que los no usuarios de Tinder utilizaban menos el preservativo. No se observan efectos de la interacción entre el uso de Tinder y el sexo. El efecto de uso de Tinder y sexo en la búsqueda de sensaciones sexuales y compulsividad sexual es significativo, y muestra como los hombres y los usuarios de Tinder puntúan más alto en dichas variables. La interacción uso de Tinder y sexo no tiene efecto alguno en la búsqueda de sensaciones sexuales y compulsividad sexual. El uso de Tinder tiene efecto sobre la variable sexo con dudas, pues los usuarios de Tinder exhibían más esta conducta, pero tener sexo con dudas no estuvo influido ni por el sexo ni por la interacción entre uso de Tinder y sexo. La interacción uso de Tinder y sexo únicamente tiene efecto en el número de parejas sexuales al mes, y los hombres no
Tabla 2. Tests de efectos entre sujetos para los factores Uso de Tinder y sexo

\begin{tabular}{|c|c|c|c|c|}
\hline Fuente & Variable & $\boldsymbol{F}$ & $p$ & $\eta^{2}$ \\
\hline \multirow[t]{6}{*}{ Uso de Tinder } & Uso del preservativo & 18.51 & $<.001$ & .067 \\
\hline & Parejas sexuales al mes & .071 & n.s. & .000 \\
\hline & Sexo con dudas & 5.718 & $<.05$ & .022 \\
\hline & Sexo no consentido & .004 & n.s. & .000 \\
\hline & Búsqueda de sensaciones sexuales & 8.029 & $<.01$ & .030 \\
\hline & Compulsividad sexual & 7.670 & $<.01$ & .029 \\
\hline \multirow[t]{6}{*}{ Sexo } & Uso del preservativo & .767 & n.s. & .003 \\
\hline & Parejas sexuales al mes & 1.639 & n.s. & .006 \\
\hline & Sexo con dudas & .765 & n.s. & .003 \\
\hline & Sexo no consentido & 1.202 & n.s. & .005 \\
\hline & Búsqueda de sensaciones sexuales & 22.177 & $<.001$ & .070 \\
\hline & Compulsividad sexual & 10.046 & $<.005$ & .037 \\
\hline \multirow{6}{*}{$\begin{array}{l}\text { Uso de Tinder } \\
\text { x sexo }\end{array}$} & Uso del preservativo & .054 & n.s. & .000 \\
\hline & Parejas sexuales al mes & 4.358 & $<.05$ & .017 \\
\hline & Sexo con dudas & .069 & n.s. & .000 \\
\hline & Sexo no consentido & .083 & n.s. & .000 \\
\hline & Búsqueda de sensaciones sexuales & .026 & n.s. & .000 \\
\hline & Compulsividad sexual & .003 & n.s. & .000 \\
\hline
\end{tabular}

usuarios de Tinder son los que mayor número de parejas sexuales tuvieron, seguidos de las mujeres usuarias de Tinder. En el resto de variables no se observan diferencias debidas a los efectos de uso de Tinder, sexo, o la combinación de estos.

Se observaron correlaciones entre edad y nivel de estudios $(r=.191)$. Se encontró además que el número de relaciones sexuales por semana se correlacionó positivamente con el número de parejas sexuales al mes $(r=.270)$, y negativamente con el uso del preservativo $(r=-.408)$. Las variables búsqueda de sensaciones sexuales y compulsividad sexual se correlacionaron positivamente entre ellas $(r=.377)$. El uso problemático de Tinder se correlacionó significativamente con compulsividad sexual, búsqueda de sensaciones sexuales y edad, así como con la frecuencia de conexión a Tinder, minutos por conexión, matches conocidos en persona y matches con los que se había tenido sexo (Tabla 3).

\section{Discusión}

Este estudio tenía por objetivos: analizar la relación entre el uso de Tinder, la búsqueda de sensaciones sexuales, la compulsividad sexual y el mantenimiento de conductas sexuales de riesgo.

Se ha observado que los usuarios de Tinder puntúan más alto en búsqueda de sensaciones sexuales y compulsividad sexual, tienen más relaciones sexuales con 
Tabla 3. Intercorrelaciones de las variables estudiadas

\begin{tabular}{|c|c|c|c|c|c|c|c|}
\hline & 1. & 2. & 3. & 4. & 5. & 6. & 7. \\
\hline 1.Uso problemático de Tinder & - & $.246^{\star \star}$ & $.298^{\star *}$ & $.414^{\star \star}$ & .493 & $.375^{\star *}$ & -.007 \\
\hline 2. Matches conocidos en persona & & - & $.472^{\star \star}$ & .040 & $.235^{\star *}$ & $.192^{*}$ & .105 \\
\hline 3. Matches con los que se ha tenido sexo & & & - & $.204^{*}$ & .096 & $.242^{\star *}$ & .151 \\
\hline 4. Minutos/conexión a Tinder & & & & - & $.429^{* *}$ & .148 & .090 \\
\hline 5. Conexiones a Tinder/día & & & & & - & $.240^{* *}$ & -.033 \\
\hline 6. Compulsividad sexual & & & & & & - & $.403^{\star *}$ \\
\hline 7. Búsqueda de sensaciones sexuales & & & & & & & - \\
\hline
\end{tabular}

dudas sobre si querer tenerlas y hacen mayor uso del preservativo.

Al presentar los usuarios de Tinder más compulsividad sexual y más búsqueda de sensaciones sexuales que los no usuarios, se confirma la primera hipótesis de la investigación. Este resultado sugiere que tener más compulsividad sexual y búsqueda de sensaciones sexuales pueden ser dos características asociadas a usar Tinder para satisfacer necesidades sexuales; así se confirma la hipótesis 1. Esto concuerda con los resultados de Sprecher y Sprecher (2009), que indican que en entornos digitales hay más apertura a encontrar pareja, en este caso sexual.

Se ha encontrado que usar Tinder no está relacionado con el número de parejas sexuales al mes, por lo que hay una menor probabilidad de la conducta sexual de riesgo "sexo con múltiples parejas". Este resultado es consistente con los hallazgos de Smith y Anderson (2016) según los cuales hasta un tercio de los usuarios de este tipo de app no había llegado a verse en persona con ningún match. Es más, se ha visto que el colectivo estudiado que tenía más parejas sexuales eran los hombres no usuarios de Tinder. En este sentido, Griffin et al. (2018) ya publicaron que las principales razones para el uso de Tinder son "por diversión" y para "conocer gente", volviéndose a romper con la creencia de que Tinder es una plataforma eminentemente sexual.

Contrariamente a lo hipotetizado inicialmente (hipótesis 2) y en la línea de los resultados de Ingram et al. (2018), los usuarios de Tinder usan más frecuentemente el preservativo que los no usuarios, y presentan así menos prevalencia en la conducta sexual de riesgo "sexo sin preservativo". Esto puede deberse a no estar en ninguna relación sentimental formal que aporte garantías de seguridad, y habla positivamente de la salud sexual de los usuarios de Tinder, pues además de no embarcarse en relaciones sexuales con un mayor número de personas que los no usuarios, toman más precauciones al usar profilaxis.

$\mathrm{Al}$ contrario que en publicaciones anteriores (Shapiro et al., 2017), respecto a "mantener relaciones sexuales sin consentimiento", tampoco existen diferencias significativas entre los usuarios y no usuarios de Tinder. No obstante, sí se observan diferencias significativas en "mantener relaciones sexuales sin estar seguro de querer tenerlas". El mayor uso del preservativo en los usuarios de Tinder se podría explicar por este fenómeno, pues estas dudas tal vez lleven a tomar mayores precauciones a la hora de tener relaciones sexuales.

No se ha podido contar con ningún grupo bien establecido de usuarios problemáticos de Tinder debido a las bajas puntuaciones en esa escala, de modo que no se ha podido validar ni refutar que los usuarios problemáticos de Tinder tengan más búsqueda de sensaciones sexuales, compulsividad sexual (aunque exista una correlación) y mantengan más conductas sexuales de riesgo que los usuarios no problemáticos (hipótesis 3). Aun así, la correlación sugiere la existencia de una relación entre el uso de dicha app, los minutos por conexión, el número de conexiones diarias, los matches conocidos en personas y los matches con los que se ha tenido relaciones sexuales. Esto plantea dudas acerca de la conveniencia de usar el modelo de las adicciones para esta conducta, en la que tal vez se caiga en el fenómeno de la sobrepatologización (Orosz et al., 2018), especialmente si consideramos que se hace con base en muestras pequeñas y de conveniencia, en lugar de utilizar población clínica, donde posiblemente escalas como la de uso problemático de Tinder serían más útiles y precisas.

A raíz de los resultados obtenidos, se descarta que el uso de Tinder suponga un riesgo para la salud de las personas jóvenes de entre 18 y 30 años, pues los usuarios no presentan más conductas sexuales de riesgo que los no usuarios. Se ha comprobado como los sujetos que lo usan han tenido más relaciones sexuales con dudas sobre si querer tenerlas, pero no se puede afirmar que se embarquen en más relaciones sexuales "peligrosas" o "abusivas" que los no usuarios. En esta línea, y al contrario de lo hipotetizado inicialmente, se podría afirmar que los usuarios de Tinder son más "precavidos" que el resto, ya que usan el preservativo con mayor frecuencia. Tales diferencias en el uso del preservativo pueden ser explicadas en algunos casos por las dudas que se presentan sobre si tener relaciones o no.

Si bien es cierto que aquellos sujetos que usan Tinder han mostrado puntuaciones mayores en búsqueda de sensaciones sexuales y compulsividad sexual, estas son dos variables no inherentemente negativas para la salud. Este hecho se podría explicar por la principal característica de Tinder: facilita encuentros interpersonales más allá del círculo social de la persona. De este modo, resulta comprensible que aquellas personas con ganas de vivir nuevas experiencias sexuales y más pensamientos de este tipo usen Tinder para satisfacer estas necesidades personales. Cabe destacar que los 
usuarios de Tinder no tienen ni más relaciones sexuales ni más parejas sexuales que los no usuarios, de modo que el uso de la app no se reduce meramente a favorecer encuentros sexuales en persona.

Así pues, el uso de Tinder no parece ser un factor de riesgo para la salud, sino una herramienta adicional para conocer personas nuevas.

\section{Conclusión}

Se concluye que el uso de Tinder no supone un riesgo para la salud y que esta $a p p$ se trata de un recurso más que ejerce de "intermediario" en la vida íntima de sus usuarios y les permite ampliar sus horizontes relacionales. Por lo tanto, Tinder se presenta como una alternativa válida para combatir las dificultades que los jóvenes puedan tener para establecer relaciones de pareja como la necesidad de conocerla dentro del grupo de amistades (Newett et al., 2017).

\section{Limitaciones y propuestas de futuro}

Una de las limitaciones de la investigación es que al tratarse de un muestreo intencional los resultados pueden haber estado influidos por la muestra a la que se ha tenido acceso, limitada a jóvenes de la zona de Barcelona. Futuros estudios podrían dirigirse a intentar generalizar estos resultados.

De cara a una mayor objetivación de las variables relativas al uso de Tinder, futuros estudios podrían usar nuevos cuestionarios o algunos preexistentes como el Facebook Intensity Scale (Orosz, Tóth-Király, \& Beáta, 2015), o el Cuestionario de adicción al sexo en jóvenes (Castro-Calvo, Ballester-Arnal, Salmerón-Sánchez, \& Gil-Llario, 2016) adaptados a las peculiaridades de Tinder.

Otra propuesta de investigación sería replicar el presente estudio comparando Tinder con otras apps de corte similar como Badoo o Happn, por ejemplo. Otro de los elementos que sería interesante incluir en investigaciones sobre Tinder es la opción de usar Tinder Plus, cuenta prémium de pago mensual que conlleva likes ilimitados y más superlikes al día, entre otras ventajas.

\section{Declaración de divulgación del autor}

El autor del presente manuscrito manifiesta que no existen intereses en conflicto y da su consentimiento a la publicación y divulgación del material del mismo.

\section{Referencias}

Ballester-Arnal, R., Gómez-Martínez, S., Gil-Llario, M. D., \& Salmerón-Sánchez, P. (2013). Sexual Compulsivity Scale: Adaptation and Validation in the Spanish Population, Journal of Sex \& Marital Therapy, 39:6, 526-540. https://doi.org/10.1080/0092623X.2012.665816

Castro-Calvo, J., Ballester-Arnal, R., Salmerón-Sánchez, P., \& Gil-Llario, M. D. (2016). Traducción y validación preliminar del cuestionario de adicción al sexo en jóvenes. International Journal of Developmental and Educational Psychology, 2, 319-328. http://dx.doi. org/10.17060/ijodaep.2016.n1.v2.207

Cohen, L. (2015). World attending in interaction: Multitasking, spatializing, narrativizing with mobile devices and Tinder. Discourse, Context and Media, 9, 46-54. http://dx.doi.org/10.1016/j.dcm.2015.08.001 Cohen, P., Kasen, S., Chen, H., Hartmark, C., \& Gordon, K. (2003). Variations in patterns of developmental transitions in the emerging adulthood period. Developmental Psychology, 39(4), 657-669. http://dx.doi. org/10.1037/0012-1649.39.4.657

Finkel, E. J., Eastwick, P. W., Karney, B. R., \& Reis, H. T. (2012). Online dating: a critical analysis from the perspective of psychological science. Psychological Science in the Public Interest, 13(1), 3-66. https://doi. org/10.1177/1529100612436522

Fox, J., \& Moreland, J. J. (2015). The dark side of social networking sites: An exploration of the relational and psychological stressors associated with Facebook use and affordances. Computers in Human Behavior, 45, 168-176. https://doi.org/10.1016/j.chb.2014.11.083

Gibbs, J. L., Ellison, N. B., \& Lai, C.-H. (2011). First comes love, then comes Google: an investigation on uncertainty reduction strategies and self-disclosure in online dating. Communication Research, 38(1), 70-100. https://doi.org/10.1177\%2F0093650210377091

Gil-Llario, M. D., Morell-Mengual, V., Ballester-Arnal, R., Giménez-García, C., \& Castro-Calvo, J. (2015). Sexual sensation seeking in Spanish young men and women with different sexual orientations. Journal of sex \& marital therapy, 41(5), 525-530.

Green, S. M., Turner, D., \& Logan, R. G. (2018). Exploring the effect of sharing common Facebook friends on the sexual risk behaviors of Tinder users. Cyberpsychology, Behavior and Social Networking, 21(7), 457462. doi: 10.1089/cyber.2017.0581.

Griffin, M., Canevello, A., \& Mcanulty, R. D. (2018). Motives and concerns associated with geosocial networking app usage: An exploratory study among heterosexual college students in the United States. Cyberpsychology, Behavior and Social Networking, 21(4), 268-275. doi: 10.1089/cyber.2017.0309.

Gullette, D. L., \& Lyons, M. A. (2006). Sensation seeking, self-esteem, and unprotected sex in college students. Journal of the Association of Nurses in AIDS Care, 17(5), 23-31.

Heino, R. D., Ellison, N. B., \& Gibbs, J. L. (2010). Relationshopping: Investigating the market metaphor in online dating. Journal of Social and Personal Relationships, 27(4), 427-447. http://dx.doi.org/10.1177/0265407510361614 Ingram, L. A., Macauda, M., Lauckner, C., \& Robillard, A. (2018). Sexual behaviors, mobile technology use and sexting among college students in the American South. American Journal of Health Promotion, 20(10), 1-10. doi: 10.1177/0890117118779008.

Jurgenson, N. (2012). When atoms meet bits: Social media, the mobile web and augmented revolution. The Future Internet, 4, 83-91. https://doi.org/10.3390/ fi4010083 
Kalichman, S. C., Johnson, J. R., Adair, V., Rompa, D., \& Kelly, J. A. (1994). Sexual sensation seeking: scale development and predicting AIDS-risk behavior among homosexually active men. Journal of Personality Assessment, 62(3), 385-397.

Murray, B. C. E., \& Campbell, E. C. (2015). The pleasures and perils of technology in intimate relationships. Journal of Couple and Relationship Therapy, 14. 116-140. https://doi.org/10.1080/15332691.2014.9 53651

Newett, L., Churchill, B., \& Robards, B. (2018). Forming connections in the digital era: Tinder, a new tool in young Australian intimate life. Journal of Sociology, 54(3), 346-361.

Orosz, G., Benyó, M., Berkes, B., Nikoletti, E., Gál, É., Tóth-Király, I., \& Bôthe, B. (2018). The personality, motivational, and need-based background of problematic Tinder use. Journal of behavioral addictions, 7(2), 301-316.

Orosz, G., Tóth-Király, I., \& Beáta, B. (2015). Four facets of Facebook intensity - The development of the Multidimensional Facebook Intensity Scale. Personality and Individual Differences, 100, 95-104. http:// dx.doi.org/10.1016/j.paid.2015.11.038

Orosz, G., Tóth-Király, I., Bôthe, B., \& Melher, D. (2016). Too many swipes for today: The development of the problematic Tinder use scale (PTUS). Journal of Behavioral Addictions, 5(3), 518-523. doi: 10.1556\%2F2006.5.2016.016

Ranzini, G., \& Lutz, C. (2017). Love at first swipe? Explaining Tinder self-presentation and motives.
Mobile Media and Communication, 5(1), 80-101.doi: $0.1177 \% 2$ F2050157916664559

Sales, N. J. (2015). Vanity Fair: Tinder and the dawn of "dating apocalypse". Recuperado de https://www. vanityfair.com/culture/2015/08/tinder-hook-upculture-end-of-dating.

Shapiro, G. K., Tatar, O., Sutton, A., Fisher, W., Naz, A., Perez, S., \& Rosberger, Z. (2017). Correlates of Tinder use and risky sexual behaviors in young adults. $C y$ berpsychology, Behavior, and Social Networking, 20(12), 727-734. doi: 10.1089/cyber.2017.0279

Smith, A. (2015). Pew Research Centre: U.S. smartphone use in 2015. Recuperado de http://www.pewinternet.org/2015/04/01/us-smartphone-use-in-2015.

Smith, A., \& Anderson, M. (2016). Pew Research Centre: 5 facts about online dating. Recuperado de http:// www.pewresearch.org/fact-tank/2016/02/29/5-factsabout-online-dating/

Sprecher, S., \& Sprecher, S. (2009). Marriage \& family review relationship initiation and formation on the Internet. Marriage \& Family Review, 45(6-8), 761-782. https://doi.org/10.1080/01494920903224350

Timmermans, E., \& De Caluwé, E. (2017a). To Tinder or not to Tinder, that's the question: An individual differences perspective to Tinder use and motives. Personality and Individual Differences, 110, 74-79. DOI: 10.1016/j.paid.2017.01.026

Timmermans, E., \& De Caluwé, E. (2017b). Development and validation of the Tinder motives scale (TMS). Computers in Human Behavior, 70, 341-350. http://dx.doi.org/10.1016/j.chb.2017.01.028 